\title{
Dirac magnon pairing via pumping
}

\author{
Vladimir A. Zyuzin $\odot$ \\ Nordita, KTH Royal Institute of Technology and Stockholm University, Roslagstullsbacken 23, SE-106 91 Stockholm, Sweden
}

(Received 10 February 2021; accepted 26 August 2021; published 21 September 2021)

\begin{abstract}
We study pumping of magnons to the Dirac points of magnon's Brillouin zone of a ferromagnet on a honeycomb lattice. In particular, we consider second-order Suhl process, when due to interaction between magnons, a pair of magnons is created due to absorption of two electromagnetic wave quanta. We introduce a bosonic analog of the Cooper ladder for the magnon pair, which is shown to enhance the pairing of magnons at the Dirac points. As a result of pairing of the Dirac magnons, the system becomes unstable towards formation of a magnetic state with zero magnetization - the Dirac magnon paired state. In this case the resonant frequency of the pump equals to that of energy of the Dirac points. Our estimates suggest that the Dirac magnon paired state can be found in the $\mathrm{CrBr}_{3}$ or $\mathrm{CrCl}_{3}$ ferromagnet below in vicinity of the Curie temperature.
\end{abstract}

DOI: 10.1103/PhysRevB.104.L100414

Magnons are fluctuations about the spontaneous magnetic order. Typically two types of magnons are distinguished based on the magnetic structure, ferromagnetic or antiferromagnetic. The two have different low-energy, low-momentum dispersion, regardless of the lattice structure of the magnetic structure. Ferromagnetic magnons are quadratic in momentum, while antiferromagnetic are linear (for example, see Refs. [1-3]). Recently, because of the progress made in topological properties of fermions, a topology tool has been applied to understand intrinsic transport properties of magnons. With that details of the lattice structure became important. Certain lattices, for example, pyrochlore [4,5], kagome [5-9], and honeycomb [10-19], allow for natural magnons's momentum-pseudospin locking. In ferromagnets such locking results in Dirac crossing points (degeneracies) at some particular high-energy and finite-momentum points in magnon's Brillouin zone. It is convenient to call magnons at such crossing points as the Dirac magnons [11]. As a result of the locking, certain types of the Dzyaloshinskii-Moriya interaction, allowed by the lattice symmetry, result in various transverse responses of magnons to the temperature gradient, such as magnon thermal Hall $[4,6,13]$ and Nernst effects [9], and to fictitious gauge fields [15], such as the magnon Hall effect.

In this Letter we find another unique Dirac magnons property revealed under the second-order Suhl magnon pumping process $[1,3,20,21]$. A single Dirac magnon cannot be created in a process of absorption of one pumping field quanta. This is because such magnon is located at nonzero momentum in the Brillouin zone, and there is no way to conserve the momentum in the process of absorption, as the experimentally relevant pumping field has a zero wave vector. However, a pair of

Published by the American Physical Society under the terms of the Creative Commons Attribution 4.0 International license. Further distribution of this work must maintain attribution to the author(s) and the published article's title, journal citation, and DOI. Funded by Bibsam. magnons with opposite momenta can be created when two pump field quanta are absorbed. Such processes are known as the second-order Suhl processes [see Fig. 1(b)]. We show that this process is not present in linearized spin-wave theory of magnons but appears when the interaction between the magnons is included to the consideration. The frequency of the pump can scan the entire Brillouin zone of the magnons, and the absorption of two magnons can happen at any frequency. However, as we show in this Letter, the pump's frequency equal to the energy of the Dirac points is the resonant due to the magnon rescattering processes of the Cooper ladder type [see Fig. 2(b)]. This is because at such frequency, the system can accommodate the largest amount of magnon pairs with opposite momenta and frequencies [see Fig. 1(b)]. For frequencies away from the Dirac points, the pairing of two magnons is parametrically weakened by the rescattering processes. The resonance corresponds to an instability of the system towards formation of a zero magnetization state. Below, we refer to such resonance as the Dirac magnons paired state. We hope Dirac magnons paired state can be experimentally observed in ferromagnets with spins on pyrochlore lattice [4], or layered kagome [8] and honeycomb lattices [17-19]. In particular, based on our estimates, we predict that it can be observed in the honeycomb $\mathrm{CrBr}_{3}$ ferromagnet [17] below in vicinity of the Curie temperature.

To demonstrate the effect, let us study a model of insulating ferromagnet in which spins of length $S$ are located on the sites of honeycomb lattice [see Fig. 1(a)]. Near neighbor spins interact with each other via ferromagnetic Heisenberg exchange interaction. Ferromagnetic order is assumed to be in the $z$ direction; this can be achieved by applying a small magnetic field in the $z$ direction. There is a pumping field which is perpendicular to the order and which oscillates with a frequency $\Omega$ and has a zero wave vector. Hamiltonian of the system reads,

$$
H=-J \sum_{\langle i j\rangle} \mathbf{S}_{i} \mathbf{S}_{j}+\Gamma \sum_{i}\left[S_{i}^{x} \cos (\Omega t)+S_{i}^{y} \sin (\Omega t)\right]
$$




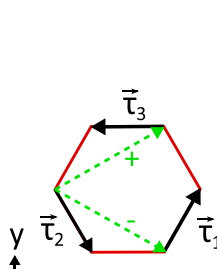

(a)

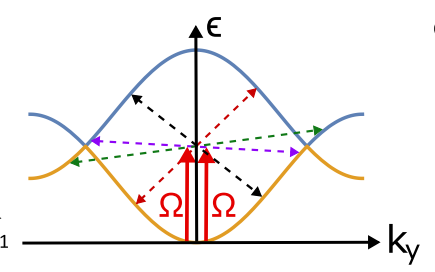

(b)

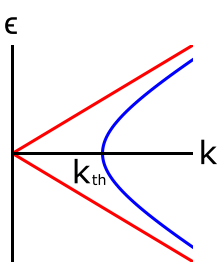

(c)
FIG. 1. (a) Schematics of the honeycomb lattice. Vectors connecting the nearest neighbor cites are $\tau_{1}=\frac{1}{2}\left(\frac{1}{\sqrt{3}}, 1\right), \tau_{2}=$ $\frac{1}{2}\left(\frac{1}{\sqrt{3}},-1\right)$, and $\boldsymbol{\tau}_{3}=\frac{1}{\sqrt{3}}(-1,0)$. (b) $k_{x}=0$ slice of spectrum of free magnons. Dashed lines describe examples of different pairs with $\mathbf{k}$, $\Omega+\epsilon$ and $-\mathbf{k}, \Omega-\epsilon$ momenta and frequency, for pump's frequency $\Omega=3 S J$. (c) Magnon spectra below in vicinity of the Dirac point: original (red) linear spectrum of free magnons and spectrum of magnon pairs (blue) with a threshold $k_{\text {th }}$ defined in Eq. (10).

where $J>0$ is the exchange coupling energy and $\Gamma$ is the pump's intensity. In order to study the spin waves, we use the Holstein-Primakoff presentation of spin operators in terms of bosons, namely for A atoms (there are two atoms in the unit cell, A and B) operators $S_{i}^{ \pm}=S_{i}^{x} \pm i S_{i}^{y}$ and $S_{i}^{z}$ read as $S_{i}^{+}=\sqrt{2 S-a_{i}^{\dagger} a_{i}} a_{i}, S_{i}^{-}=a_{i}^{\dagger} \sqrt{2 S-a_{i}^{\dagger} a_{i}}$, and $S_{i}^{z}=S-a_{i}^{\dagger} a_{i}$, with $\left[a_{i}, a_{j}^{\dagger}\right]=\delta_{i, j}$ boson commutation relation. The same is performed for the $\mathrm{B}$ atoms with the help of $b_{i}$ and $b_{i}^{\dagger}$ boson operators.

In the space of elements of the honeycomb's unit cell, in which case the boson operators are defined by $\Psi_{\mathbf{k}}^{\dagger}=\left(a_{\mathbf{k}}^{\dagger}, b_{\mathbf{k}}^{\dagger}\right)$, the Hamiltonian of noninteracting spin waves reads as

$$
H_{0}=S J \int_{\mathbf{k}} \Psi_{\mathbf{k}}^{\dagger}\left[\begin{array}{cc}
3 & -\gamma_{\mathbf{k}} \\
-\gamma_{\mathbf{k}}^{*} & 3
\end{array}\right] \Psi_{\mathbf{k}} \equiv \int_{\mathbf{k}} \Psi_{\mathbf{k}}^{\dagger}\left[\hat{H}_{0}\right]_{\mathbf{k}} \Psi_{\mathbf{k}}
$$

where $\gamma_{\mathbf{k}}=\sum_{i=1,2,3} e^{i \mathbf{k} \tau_{i}}=2 e^{i \frac{k_{x}}{2 \sqrt{3}}} \cos \left(\frac{k_{y}}{2}\right)+e^{-i \frac{k x}{\sqrt{3}}}$ is the nearest-neighbor hopping element see the left part of Fig. 1, and $\int_{\mathbf{k}} \equiv \int \frac{d \mathbf{k}}{(2 \pi)^{2}}$ for the two-dimensional system. Diagonalization gives energy spectrum of noninteracting magnons, $\varepsilon_{ \pm ; \mathbf{k}}=S J\left(3 \pm\left|\gamma_{\mathbf{k}}\right|\right)$ with corresponding wave functions $\varphi_{ \pm}=$ $\frac{1}{\sqrt{2}}\left[\mp \frac{\gamma_{\mathbf{k}}}{\mid \gamma_{\mathbf{k}}}, 1\right]^{\mathrm{T}}$. At special $\mathbf{K}=\left(0,-\frac{4 \pi}{3}\right)$ and $\mathbf{K}^{\prime}=\left(0, \frac{4 \pi}{3}\right)$ points the spectrum is linear and is described by the Dirac Hamiltonian. The energy of magnons at these points is $\varepsilon_{ \pm ; \mathbf{k}}=$ $3 S J$. Terms quartic in boson operators describe interactions between the magnons. Normal ordered interaction reads

$$
\begin{aligned}
H_{\text {int }}= & -J \int_{\{\mathbf{k}\}} \delta_{\{\mathbf{k}\}} \gamma_{\mathbf{k}_{4}-\mathbf{k}_{3}} a_{\mathbf{k}_{1}}^{\dagger} b_{\mathbf{k}_{3}}^{\dagger} a_{\mathbf{k}_{2}} b_{\mathbf{k}_{4}} \\
& +\frac{J}{4} \int_{\{\mathbf{k}\}} \delta_{\{\mathbf{k}\}}\left[\gamma_{\mathbf{k}_{3}}^{*} a_{\mathbf{k}_{1}}^{\dagger} b_{\mathbf{k}_{3}}^{\dagger} a_{\mathbf{k}_{2}} a_{\mathbf{k}_{4}}+\gamma_{\mathbf{k}_{3}} a_{\mathbf{k}_{2}}^{\dagger} a_{\mathbf{k}_{4}}^{\dagger} a_{\mathbf{k}_{1}} b_{\mathbf{k}_{3}}\right] \\
& +\frac{J}{4} \int_{\{\mathbf{k}\}} \delta_{\{\mathbf{k}\}}\left[\gamma_{\mathbf{k}_{1}} a_{\mathbf{k}_{1}}^{\dagger} b_{\mathbf{k}_{3}}^{\dagger} b_{\mathbf{k}_{2}} b_{\mathbf{k}_{4}}+\gamma_{\mathbf{k}_{1}}^{*} b_{\mathbf{k}_{2}}^{\dagger} b_{\mathbf{k}_{4}}^{\dagger} a_{\mathbf{k}_{1}} b_{\mathbf{k}_{3}}\right]
\end{aligned}
$$

where $\delta_{\{\mathbf{k}\}} \equiv \delta_{\mathbf{k}_{1}-\mathbf{k}_{2}, \mathbf{k}_{4}-\mathbf{k}_{3}}$ short notation was used, and $\int_{\{\mathbf{k}\}}$ stands for integration over all momenta. The interaction is instantaneous in time. Hamiltonian in momentum space de- scribing pump field with a frequency $\Omega$ is

$$
H_{\text {pump }}=\frac{\Gamma \sqrt{S}}{\sqrt{2}}\left[\left(a_{0}+b_{0}\right) e^{-i \Omega t}+\left(a_{0}^{\dagger}+b_{0}^{\dagger}\right) e^{i \Omega t}\right],
$$

where $a_{0} \equiv a_{\mathbf{k}=0}$ and the same for $b_{0}$. In order to understand the effect of the pumping field Eq. (4) on the magnons described by Eqs. (2) and (3), we study the system in the Keldysh time space. This space complicates the analysis but gives a clear understanding of all relevant processes. We promote boson fields $a_{\mathbf{k}}, b_{\mathbf{k}}$ to $\Psi_{\alpha ; \mathbf{k} ; \epsilon}, \Psi_{\beta ; \mathbf{k} ; \epsilon}$ fields, in which frequency $\epsilon$ was explicitly used, and $a_{\mathbf{k}}^{\dagger}, b_{\mathbf{k}}^{\dagger}$ to $\bar{\Psi}_{\alpha ; \mathbf{k} ; \epsilon}, \bar{\Psi}_{\beta ; \mathbf{k} ; \epsilon}$. Furthermore, the fields are promoted to classical (cl) and quantum components (q) components in accord with the Keldysh technique (see Supplemental Material [22] for details and, for example, Ref. [23]). Let us show how to conveniently capture the process of absorption of the pumping field Eq. (4) by the magnons.

We write the advanced part of the Lagrangian describing noninteracting magnons defined by Eq. (2) with $\epsilon=\Omega$ frequency and $\mathbf{k}=0$ momentum,

$$
\mathcal{L}_{0, \Omega}^{\mathrm{A}}=\sum_{m, n} \bar{\Psi}_{m, 0, \Omega}^{\mathrm{cl}} \hat{\mathcal{L}}_{m n, 0, \Omega}^{\mathrm{A}} \Psi_{n, 0, \Omega}^{\mathrm{q}}-\Gamma \sqrt{S} \sum_{n} \Psi_{n, 0, \Omega}^{\mathrm{q}},
$$

where $\hat{\mathcal{L}}_{m n, \mathbf{k}, \Omega}^{\mathrm{A}}=(\Omega-i 0) \delta_{m n}-\left[\hat{H}_{0}\right]_{m n, \mathbf{k}}$ is the Lagrangian density describing corresponding noninteracting magnons, and $m, n=\{\alpha, \beta\}$ are the indeces describing pseudospin. We now want to get rid of the terms linear in $\Psi_{n, 0, \Omega}^{\mathrm{q}}$ in the action with the following shift,

$$
\bar{\Psi}_{\alpha, 0, \Omega}^{\mathrm{cl}} \rightarrow \bar{\Psi}_{\alpha, 0, \Omega}^{\mathrm{cl}}+x_{\mathrm{A}}, \quad \bar{\Psi}_{\beta, 0, \Omega}^{\mathrm{cl}} \rightarrow \bar{\Psi}_{\beta, 0, \Omega}^{\mathrm{cl}}+y_{\mathrm{A}},
$$

where $x_{\mathrm{A}}$ and $y_{\mathrm{A}}$ are obtained to be

$$
\begin{aligned}
x_{\mathrm{A}} & =\frac{\mathcal{L}_{\beta \alpha, 0, \Omega}^{\mathrm{A}}-\mathcal{L}_{\beta \beta, 0, \Omega}^{\mathrm{A}}}{\mathcal{L}_{\alpha \beta, 0, \Omega}^{\mathrm{A}} \mathcal{L}_{\beta \alpha, 0, \Omega}^{\mathrm{A}}-\mathcal{L}_{\beta \beta, 0, \Omega}^{\mathrm{A}} \mathcal{L}_{\alpha \alpha, 0, \Omega}^{\mathrm{A}}} \Gamma \sqrt{S}, \\
y_{\mathrm{A}} & =\frac{\mathcal{L}_{\alpha \beta, 0, \Omega}^{\mathrm{A}}-\mathcal{L}_{\alpha \alpha, 0, \Omega}^{\mathrm{A}}}{\mathcal{L}_{\alpha \beta, 0, \Omega}^{\mathrm{A}} \mathcal{L}_{\beta \alpha, 0, \Omega}^{\mathrm{A}}-\mathcal{L}_{\beta \beta, 0, \Omega}^{\mathrm{A}} \mathcal{L}_{\alpha \alpha, 0, \Omega}^{\mathrm{A}}} \Gamma \sqrt{S} .
\end{aligned}
$$

The same procedure is performed for the retarded part of the action to take care of the $-\Gamma \sqrt{S} \sum_{n} \bar{\Psi}_{n, 0, \Omega}^{\mathrm{q}}$ linear term. See Ref. [22] for more details. After the shifts are performed, the noninteracting action is the same as the one without the linear terms. Even the Keldysh part of the action does not get affected. However, the shifts drastically modify terms describing magnon-magnon interactions Eq. (3). In particular, new terms in the bilinear part of the Hamiltonian describing magnons with any frequency and momentum, rather than the pumped ones with $\Omega$ and $\mathbf{k}=0$, appear. Our calculations show (see Ref. [22] for details) that there is no way to obtain terms of the $\propto \bar{\Psi}_{n, \mathbf{k}, \epsilon}^{\mathrm{cl} / \mathrm{q}} \Psi_{m, \mathbf{k}, \epsilon}^{\mathrm{q} / \mathrm{cl}}$ type, but new terms describing pairing [20] of two magnons, i.e., of the $\propto \bar{\Psi}_{n, \mathbf{k}, \Omega+\epsilon}^{\mathrm{cl} / \mathrm{q}} \bar{\Psi}_{m,-\mathbf{k}, \Omega-\epsilon}^{\mathrm{q} / \mathrm{cl}}$ type, appear. Physically, they originate due to the absorbtion of two pump's quanta and describe a subsequent creation of a magnon pair with $\mathbf{k}, \Omega+\epsilon$ and $-\mathbf{k}, \Omega-\epsilon$ momenta and energies [see Fig. 1(b)].

Let us now understand what will the creation of a magnon pair do to the system. Our calculations show that in the extended space of magnons, $\bar{\Phi}_{\mathbf{k}, \epsilon}^{\mathrm{cl} / \mathrm{q}}=$ $\frac{1}{\sqrt{2}}\left(\bar{\Psi}_{\alpha, \mathbf{k}, \Omega+\epsilon}^{\mathrm{cl} / \mathrm{q}}, \bar{\Psi}_{\beta, \mathbf{k}, \Omega+\epsilon}^{\mathrm{cl} / \mathrm{q}}, \Psi_{\alpha,-\mathbf{k}, \Omega-\epsilon}^{\mathrm{cl} / \mathrm{q}}, \Psi_{\beta,-\mathbf{k}, \Omega-\epsilon}^{\mathrm{cl} / \mathrm{q}}\right)$, the spectrum 


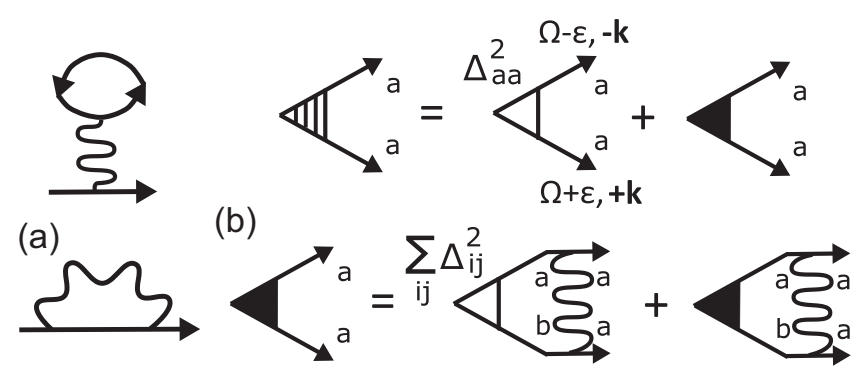

FIG. 2. (a) Hartree-Fock corrections to the dispersion of magnons. Wavy lines stand for the interaction defined in Eq. (3). (b) Graphic equation for the pairing interaction strength. Here empty triangle stands for the initial pairing interaction strength $\Delta_{i j}^{2}$ defined in accordance with Eq. (9), $\Delta_{\mathrm{aa}}^{2}=\Delta_{\mathrm{bb}}^{2}=-\Delta^{2} \gamma_{0}$, and $\Delta_{\mathrm{ab}}^{2}=$ $\left(\Delta_{\mathrm{ba}}^{2}\right)^{*}=\Delta^{2} \gamma_{\mathbf{k}}$. Black triangle is intermediately renormalized pairing interaction strength, and lined triangle is the overall renormalized pairing interaction strength.

of a pair of magnons is given by a solution of the following secular equation,

$$
\operatorname{det}\left[\begin{array}{cccc}
\zeta+\epsilon & S J \gamma_{\mathbf{k}} & -\Delta^{2} \gamma_{0} & \Delta^{2} \gamma_{\mathbf{k}} \\
S J \gamma_{\mathbf{k}}^{*} & \zeta+\epsilon & \Delta^{2} \gamma_{\mathbf{k}}^{*} & -\Delta^{2} \gamma_{0} \\
-\Delta^{2} \gamma_{0} & \Delta^{2} \gamma_{\mathbf{k}} & \zeta-\epsilon & S J \gamma_{\mathbf{k}} \\
\Delta^{2} \gamma_{\mathbf{k}}^{*} & -\Delta^{2} \gamma_{0} & S J \gamma_{\mathbf{k}}^{*} & \zeta-\epsilon
\end{array}\right]=0,
$$

where $\zeta=\Omega-3 S J$ is introduced for brevity, $\gamma_{0}=3$, and where the pairing strength $\Delta^{2}=\frac{J}{4}\left(\frac{\Gamma \sqrt{S}}{3 S J}\right)^{2}$ for $\zeta=0$, and $\Delta^{2}=\frac{J}{4}\left(\frac{\Gamma \sqrt{S}}{6 S J}\right)^{2}$ for $\zeta= \pm 3 S J$ and otherwise according to the shift Eq. (6), was defined. The equation is the boson analog of the Bogoliubov-de Gennes Hamiltonian in fermion systems. The difference is in the structure of signs of the frequencies $\epsilon$ on the main diagonal in Eq. (9). The spectrum reads

$$
\epsilon_{ \pm ; \mathbf{k}}^{2}=\left(\zeta \pm S J\left|\gamma_{\mathbf{k}}\right|\right)^{2}-\Delta^{4}\left(\gamma_{0} \mp\left|\gamma_{\mathbf{k}}\right|\right)^{2}
$$

Therefore, the system of pumped interacting magnons will become unstable when $\epsilon_{ \pm ; \mathbf{k}}^{2}<0$ is satisfied. Let us analyze different parts of the spectrum for such an instability.

Let us first study a special case when pump's frequency is $\Omega=3 S J$ for which $\zeta=0$. Then, at the $\Gamma=(0,0)$ point $\left|\gamma_{\mathbf{k}}\right| \approx 3-\frac{k^{2}}{4}$, then $\epsilon_{+; \mathbf{k}}^{2}=(S J)^{2}\left(3-\frac{k^{2}}{4}\right)^{2}-36 \Delta^{4}$. For the instability to occur at the $\Gamma$ point, the intensity of the pump should become larger than the exchange coupling energy. However, experimentally reasonable assumption is $S J>\Delta^{2}$ which means it is impossible to make the system unstable at the $\boldsymbol{\Gamma}$ point. On the other hand, at the $\mathbf{K}$ and $\mathbf{K}^{\prime}$ we approximate $\left|\gamma_{\mathbf{k}}\right| \approx \frac{\sqrt{3}}{2} k$ and get for the spectrum $\epsilon_{ \pm ; \mathbf{k}}^{2} \approx$ $(S J)^{2} \frac{3}{4} k^{2}-9 \Delta^{4}$. From here we observe that the solution is always unstable for momenta smaller than the threshold value of $k_{\mathrm{th}}=\frac{2 \sqrt{3} \Delta^{2}}{S J}$, i.e., for $k<k_{\mathrm{th}}$. For schematics see Fig. 1(c).

Having pumped the magnons to the Dirac points, let us now study their rescattering processes. In the first order in interaction Eq. (3) we get Hartree-Fock type corrections shown in Fig. 2(a) to the magnon's dispersion [24]. See Ref. [22] for details of their derivation. Interaction Eq. (3) treated to second order contributes to the magnon's lifetime [16]. Here we study how the pairing interaction strength $\Delta^{2}$ gets renormalized by the interaction. For that we construct a boson analog of the Cooper ladder shown in Fig. 2(b). Our calculations show (see
Ref. [22] for details) that the operator structure of $\Delta^{2}$ given in Eq. (9) gets reproduced at each step of the ladder. Then, summing up the ladder, we replace $\Delta^{2}$ for $\zeta=0$ with

$$
\Delta^{2} \rightarrow \frac{\Delta^{2}}{1-\frac{J}{\tilde{J}}\left[\frac{1}{4 S}+\frac{\pi}{8 S}\left(\frac{T}{3 S \tilde{J}}\right)^{2}\right]},
$$

where $\tilde{J}=J\left[1-\frac{\pi}{4 S}\left(\frac{T}{3 S J}\right)^{2}\right]$ includes the Hartree-Fock corrections. The integral defining a step of the ladder is counting the number of pairs which can be created for a given frequency. Clearly the pairing of Dirac magnons is enhanced due to the rescattering processes. The minus sign in the denominator in Eq. (11) is due to the repulsive nature of the last two terms in Eq. (3). Our estimates suggest that for honeycomb lattice $\mathrm{CrBr}_{3}$ or $\mathrm{CrCl}_{3} S=\frac{3}{2}$ ferromagnet [17], the tendency is such that at temperatures below in vicinity of the Curie temperature, i.e., $T \simeq T_{c} \sim 3 S J$, the expression for the renormalized pairing strength Eq. (11) diverges. This signals a transition to a new state, which we call Dirac magnons paired state. It seems natural that the transition occurs below in the vicinity of the Curie temperature, as there are plenty of magnons in the system and their rescattering processes are known to become important [16,17,24]. If the spin is made more classical by increasing its length $S$, the denominator in Eq. (11) does not become singular for any temperature.

Let us study the effect of Dzyaloshinskii-Moriya interaction of the $H_{\mathrm{DMI}}=D \sum_{\langle\langle i j\rangle\rangle} \nu_{i j}\left[\mathbf{S}_{i} \times \mathbf{S}_{j}\right]_{z}$ type on the pairing. Here $D$ is a constant, $\langle\langle i j\rangle\rangle$ notation counts second-nearest neighbors, and $v_{i j}= \pm 1$ is defined by the green dashed arrows in Fig. 1(a) (see Ref. [22] for more details). In the vicinity of the Dirac points, i.e., $\zeta=0$, the spectrum of magnon pairs is now

$$
\epsilon_{ \pm ; \mathbf{k}}^{2}=(S J)^{2} \frac{3}{4} k^{2}+\chi^{2}-9 \Delta^{4},
$$

where $\chi=3 \sqrt{3} S D$. We conclude that if $|\chi| \geqslant 3 \Delta^{2}$ there will be no instability in the system. In unpumped ferromagnet such Dzyaloshinskii-Moriya interaction opens up a gap at the Dirac points in the spectrum of the magnons. Then, for the Dirac magnons paired state to occur, pumping strength should overcome this gap.

When $\zeta<0$ only $\epsilon_{+; \mathbf{k}}^{2}$ can become less than zero and cause instability of the system. For example, close to the $\Gamma$ point, we expand $\left|\gamma_{\mathbf{k}}\right| \approx 3-\frac{k^{2}}{4}$ and obtain for the threshold $k_{\mathrm{th}}=$ $\sqrt{\frac{\Omega}{S J}} \frac{\Delta^{2}}{S J}$ of the instability. When $3 S J>\zeta>0$ only $\epsilon_{-; \mathbf{k}}^{2}$ can become less than zero. Performing the same approximations as for the $\zeta<0$ case, we get for the threshold value of the momentum $k_{\mathrm{th}}=\frac{6 \Delta^{2}}{\sqrt{S J(3 S J-\zeta)}}$ for $3 S J-\zeta>\frac{3}{2} \Delta^{2}$, i.e., away from the $\Gamma$ point, and $k_{\mathrm{th}}=\frac{2 \sqrt{6} \Delta}{\sqrt{S J}}$ for $3 S J-\zeta<\frac{3}{2} \Delta^{2}$-in the vicinity of the $\Gamma$ point. Rescattering processes shown in Fig. 2(b) result for the $3 S J>\zeta>0$ case in

$$
\Delta^{2} \rightarrow \frac{\Delta^{2}}{1+\frac{3}{16 \pi S} \ln \left(\frac{S J \Lambda^{2}}{4(3 S J-\zeta)}\right)+i \frac{3}{16 S}},
$$

where $\Lambda$ is the high-frequency cutoff. Therefore, as $\Omega \rightarrow$ $6 S J$, the pairing of magnons vanishes. We think that this might be natural, as the one pump quanta absorption is the most effective at $\Omega=6 S J$, and the two pump quanta absorption channel must thus get closed. 
In addition to studied rescattering processes, one needs to include magnon decay rate, which originates due to interactions in second-order perturbation theory, to the main diagonal in the secular equation (9). Then the threshold value is going to be decreased by the decay rate. In particular, Ref. [16] showed (see Fig. 2 there) that the decay rate, which is $\frac{1}{\tau} \propto T^{2}$, for a honeycomb lattice ferromagnet is the smallest for the Dirac magnons and is the largest for the $\varepsilon_{+; \mathbf{k}}$ magnons in the vicinity of the $\Gamma$ point. Therefore, the threshold value for the Dirac magnon paired state instability does not get drastically modified by the decay rate. All in all, from Eq. (11) and discussions above we conclude that the Dirac magnon paired state can become the most unstable for temperatures below in the vicinity of the Curie temperature.

There are two corollaries which can be made on the nature of the Dirac magnon paired state. First of all, when the conditions for the instability are met [divergence of Eq. (11)], there is going to be an absorption peak at frequency equal to $\Omega=3 S J$ corresponding to the Dirac magnon paired state. If the system is finite and isolated, the exponential growth of the Dirac magnons pairs in time cannot last forever, and it will be stopped by interactions between the magnons, effects which are beyond studied in the present Letter. Secondly, it can be deduced that the Dirac magnon paired state is the instability of a ferromagnet towards formation of a zero magnetization state. The energy to flip one spin in the unit cell equals $6 S^{2} \mathrm{~J}$, and absorption of a pair of Dirac magnons corresponds to $6 S J$ energy. Then, for integer spins it is possible to flip the spin by creating an appropriate number of Dirac magnon pairs. For example, for $S=1$ absorption of one pair of Dirac magnons flips the spin, for $S=2$ it is four pairs of Dirac magnons, and so on. Similar argument applies to half-odd-integer spins. To start thinking about such a state, one can imagine dynamically generated antiferromagnetic order on the honeycomb lattice. However, such antiferromagnetic order will be fluctuating in time between different configurations with zero magnetization. Unlike in the experiment [25], we restrain ourselves from calling the Dirac magnon paired state as the Bose-Einstein condensate (BEC) of Dirac magnons, instead, we hypothesize that it is a condensate of Dirac magnon pairs. Detailed understanding of the nature of the new state is a question for future research.
In passing, let us discuss another possibility of pumping the magnons. First note that in the honeycomb lattice there are two energy branches at the $\Gamma$ point corresponding to $\varepsilon_{+; 0}=6 S J$ and $\varepsilon_{-; 0}=0$, which are connected by $\Omega=6 S J$ frequency. Therefore, one can excite a single magnon by a pump Eq. (4) with a frequency via a $\varepsilon_{-; 0}+\Omega \rightarrow \varepsilon_{+; 0}$ process. Pumping a single magnon will not make the system unstable in a sense of Eqs. (9) and (10). However, an additional rescattering of the excited magnon with frequency $\varepsilon_{+; 0}$ in to a pair of Dirac magnons, via $\varepsilon_{+; 0}+\varepsilon_{-; 0} \rightarrow \varepsilon_{+; \mathbf{K}}+\varepsilon_{-; \mathbf{K}^{\prime}}$ (schematically) processes, might create the Dirac magnons paired state and may cause an instability in the system. This pumping scheme is the parametric pumping similar to the one in the experiment [25]. It is possible that the Dirac magnon paired state is also going to occur in such a pumping scheme. However, it is going to coexist with unpaired magnons at $\varepsilon_{+; \mathbf{k}}=6 S J$ and $\varepsilon_{-; \mathbf{k}}=0$ energy. This is another question for future research.

To conclude, we studied second-order Suhl processes in a honeycomb ferromagnet and showed that under certain conditions the resonant pump's frequency corresponds to the energy of the Dirac points, causing an instability of the ferromagnet. This is because, as is schematically shown in Fig. 1(b), the system can accommodate the largest amount of magnon pairs, and their rescattering processes of the Cooper ladder type shown in Fig. 2(b) result in a pole structure Eq. (11), which can become singular as a function of temperature. We deduced that the instability is towards formation of a zero magnetization state and called it as the Dirac magnons paired state. We estimated that the $\mathrm{CrBr}_{3}$ or $\mathrm{CrCl}_{3}$ ferromagnet might show this Dirac magnon paired state below in the vicinity of the Curie temperature.

The author thanks A.M. Finkel'stein and A.Yu. Zyuzin for helpful discussions and to Pirinem School of Theoretical Physics for hospitality. This work was started by the author in a research group of A.V. Balatsky in Nordita, whom the author thanks for discussions. This work is supported by the VILLUM FONDEN via the Centre of Excellence for Dirac Materials (Grant No. 11744), the European Research Council under the European Unions Seventh Framework Program Synergy HERO, and the Knut and Alice Wallenberg Foundation KAW.
[1] A. I. Akhiezer, V. G. Bar'yakhtar, and S. V. Peletminskii, Spin Waves (Nauka, Moscow, in Russian, 1967).

[2] A. Auerbach, Interacting Electrons and Quantum Magnetism (Springer, New York, 1994).

[3] S. M. Rezende, Fundamentals of Magnonics (Springer, Cham, 2020).

[4] Y. Onose, T. Ideue, H. Katsura, Y. Shiomi, N. Nagaosa, and Y. Tokura, Science 329, 297 (2010).

[5] H. Du, W. Ning, M. Tian, and Y. Zhang, Phys. Rev. B 87, 014401 (2013).

[6] H. Katsura, N. Nagaosa, and P. A. Lee, Phys. Rev. Lett. 104, 066403 (2010).

[7] A. Mook, J. Henk, and I. Mertig, Phys. Rev. B 89, 134409 (2014)
[8] M. Hirschberger, R. Chisnell, Y. S. Lee, and N. P. Ong, Phys. Rev. Lett. 115, 106603 (2015).

[9] A. A. Kovalev and V. A. Zyuzin, Phys. Rev. B 93, 161106(R) (2016).

[10] P. A. Maksimov and A. L. Chernyshev, Phys. Rev. B 93, 014418 (2016).

[11] J. Fransson, A. M. Black-Schaffer, and A. V. Balatsky, Phys. Rev. B 94, 075401 (2016).

[12] S. A. Owerre, J. Phys.: Condens. Matter 28, 386001 (2016).

[13] S. A. Owerre, J. Appl. Phys. 120, 043903 (2016).

[14] S. K. Kim, H. Ochoa, R. Zarzuela, and Y. Tserkovnyak, Phys. Rev. Lett. 117, 227201 (2016).

[15] A. A. Kovalev, V. A. Zyuzin, and B. Li, Phys. Rev. B 95, 165106 (2017) 
[16] S. S. Pershoguba, S. Banerjee, J. C. Lashley, J. Park, H. Ågren, G. Aeppli, and A. V. Balatsky, Phys. Rev. X 8, 011010 (2018).

[17] W. B. Yelon and R. Silberglitt, Phys. Rev. B 4, 2280 (1971).

[18] L. Chen, J.-H. Chung, B. Gao, T. Chen, M. B. Stone, A. I. Kolesnikov, Q. Huang, and P. Dai, Phys. Rev. X 8, 041028 (2018).

[19] B. Yuan, I. Khait, G.-J. Shu, F. C. Chou, M. B. Stone, J. P. Clancy, A. Paramekanti, and Y.-J. Kim, Phys. Rev. X 10, 011062 (2020).
[20] H. Suhl, J. Phys. Chem. Solids 1, 209 (1957).

[21] V. E. Zakharov, V. S. L'vov, and S. S. Starobinets, Sov. Phys. JETP 32, 656 (1971).

[22] See Supplemental Material at http://link.aps.org/supplemental/ 10.1103/PhysRevB.104.L100414 for details of derivations.

[23] A. Kamenev, Field Theory of Non-Equilibrium Systems (Cambridge University Press, Cambridge, 2012).

[24] M. Bloch, Phys. Rev. Lett. 9, 286 (1962).

[25] S. O. Demokritov, V. E. Demidov, O. Dzyapko, G. A. Melkov, A. A. Serga, B. Hillebrands, and A. N. Slavin, Nature (London) 443, 430 (2006). 